\title{
SHARP BOUNDS ON THE EXPECTATIONS OF LINEAR COMBINATIONS OF $k$ th RECORDS EXPRESSED IN THE GINI MEAN DIFFERENCE UNITS
}

\author{
BY
}

PAWEL MARCIN KOZYRA (WARSZAWA) AND TOMASZ RYCHLIK* (WARSZAWA)

\begin{abstract}
We describe a method of calculating sharp lower and upper bounds on the expectations of linear combinations of $k$ th records expressed in the Gini mean difference units of the original i.i.d. observations. In particular, we provide sharp lower and upper bounds on the expectations of $k$ th records and their differences. We also present the families of distributions which attain the bounds in the limit.
\end{abstract}

2010 AMS Mathematics Subject Classification: Primary: 60E15, $62 \mathrm{G} 32$.

Key words and phrases: Expectation, Gini mean difference, sharp bound, $k$ th record.

\section{INTRODUCTION AND MAIN RESULT}

Let $X_{1}, X_{2}, \ldots$ be i.i.d. random variables with common continuous cumulative distribution function $F$. Assume that $X_{i: n}$ stands for the $i$ th order statistic obtained from the first $n$ observations. For a given $k \in \mathbb{N}$, the sequence of $k$ th (upper) record values $\left(R_{n, k}: n \in \mathbb{N}\right)$ based on the sequence $\left(X_{n}: n \in \mathbb{N}\right)$ was defined by Dziubdziela and Kopociński [ []] as $R_{n, k}=X_{T_{n, k}: T_{n, k}+k-1}$, where $T_{1, k}=1$ and $T_{n+1, k}=\min \left\{j>T_{n, k}: X_{j: j+k-1}>X_{T_{n, k}: T_{n, k}+k-1}\right\}, n \in \mathbb{N}$, are the respective occurrence times of $k$ th records. This is a generalization of the notion of classic upper records with $k=1$ introduced by Chandler [3]. In this section, we determine sharp lower and upper bounds for expectations of arbitrary linear combinations of $k$ th records $\mathbb{E}\left[\sum_{i=1}^{n} c_{i}\left(R_{i, k}-\mu\right)\right]$, centered about the population mean $\mu=\mathbb{E} X_{1}$, and expressed in the Gini mean difference units $\Delta=\mathbb{E}\left|X_{1}-X_{2}\right|$. In Section 2, we specify the bounds on the expectations of centered $k$ th records $R_{n, k}-\mu$ and their differences $R_{n, k}-R_{m, k}$. Section 3 contains the proofs of the results presented in Section 2.

* The second author was supported by the National Science Centre, Poland, grant no. 2015/19/B/ST1/03100. 
The Gini mean difference is a simple useful measure of dispersion. For defining it, only finiteness of the first population moment is sufficient. By now, various evaluations of $\mathbb{E}\left[\sum_{i=1}^{n} c_{i}\left(R_{i, k}-\mu\right)\right]$ for specific $\underline{c}=\left(c_{1}, \ldots, c_{n}\right)$ were presented in terms of scale units $\sigma_{p}=\left[\mathbb{E}\left|X_{1}-\mu\right|^{p}\right]^{1 / p}$ generated by $p$ th absolute central moments. The first result of this type was presented by Nagaraja [16] who applied the Schwarz inequality for getting sharp bounds on the expectations of the classic record values expressed in terms of the mean $\mu$ and standard deviation $\sigma_{2}$ of the parent distribution. Raqab [19] used the Hölder inequality in order to receive bounds expressed in terms of other scale units $\sigma_{p}, p \geqslant 1$. He also derived refined estimates of the records coming from symmetric populations. Rychlik [24] evaluated the expectations of record spacings $\mathbb{E}\left(R_{n, 1}-R_{n-1,1}\right)$ in the general populations as well as under the restrictions to the distributions with increasing density and increasing failure rate. Danielak [4] generalized these results to arbitrary record increments $\mathbb{E}\left(R_{n, 1}-R_{m, 1}\right), n>m$.

For general $k$ th records, Grudzień and Szynal [10] obtained non-optimal evaluations in terms of $\mu$ and $\sigma_{2}$ by direct use of the Schwarz inequality. Raqab [18] applied a modification of the Schwarz inequality proposed by Moriguti [15] in order to get optimal bounds. Raqab and Rychlik [22] used both the Moriguti and Hölder inequalities and calculated the bounds measured in various $\sigma_{p}$ units. Similar results for the differences of adjacent and non-adjacent $k$ th records were derived by Raqab [20], and Danielak and Raqab [5], respectively. Goroncy and Rychlik [9] determined the lower bounds on the expectations of centered values of $k$ th records, and their differences expressed in $\sigma_{p}$ units.

Raqab and Rychlik [23] calculated optimal evaluations for the second record values coming from symmetric populations. Gajek and Okolewski [8] provided the sharp bounds on the expectations of $k$ th records coming from the decreasing density and failure rate populations expressed in the population second raw moments. Optimal mean-variance inequalities for the expected $k$ th record spacings from the above models were presented in Danielak and Raqab [6]. Second record non-adjacent differences coming from populations with decreasing density functions were studied in Raqab [21]. Tight upper bounds for the $k$ th record values from the decreasing generalized failure rate populations were established by Bieniek [2]. Klimczak [13] calculated sharp bounds on the expectations of $k$ th records and their differences coming from bounded populations. They were expressed in the scale units amounting to the lengths of the population support intervals.

The distribution function of the $n$th value of the $k$ th record coming from the standard uniform distribution is of the form

$$
G_{n, k}(u)=1-(1-u)^{k} \sum_{i=0}^{n-1} \frac{[-k \ln (1-u)]^{i}}{i !},
$$

where $k, n \in \mathbb{N}$ and $u \in(0,1)$. If $X_{1}, X_{2}, \ldots$ have a continuous distribution function $F$, then the composition $G_{n, k} \circ F$ is the distribution function of the $n$th value 
of the $k$ th record. In the sequel we use the following notions:

$$
\begin{aligned}
& \psi_{n, k}(u)=(1-u)^{k-1} \sum_{i=0}^{n-1} \frac{[-k \ln (1-u)]^{i}}{i !}-1, \\
& \Psi_{n, k}(u)=\frac{\psi_{n, k}(u)}{2 u}, \\
& \psi_{\underline{c}, k}(u)=\sum_{i=1}^{n} c_{i} \psi_{i, k}(u)=(1-u)^{k-1} \sum_{i=0}^{n-1} b_{i+1} \frac{[-k \ln (1-u)]^{i}}{i !}-b_{1}, \\
& \Psi_{\underline{c}, k}(u)=\sum_{i=1}^{n} c_{i} \Psi_{i, k}(u)=\frac{\psi_{\underline{c}, k}(u)}{2 u},
\end{aligned}
$$

where $\underline{c}=\left(c_{1}, \ldots, c_{n}\right) \in \mathbb{R}^{n}$, and $b_{i}=\sum_{j=i}^{n} c_{j}, i=1, \ldots, n$.

THEOREM 1.1. Let $X_{1}, X_{2}, \ldots$ be an i.i.d. sequence with a common continuous distribution function, expectation $\mu=\mathbb{E} X_{1} \in \mathbb{R}$, and Gini mean difference $\Delta=\mathbb{E}\left|X_{1}-X_{2}\right|$. Let $R_{1, k}, R_{2, k}, \ldots$ denote the respective sequence of $k$ th upper records, and assume that $\mathbb{E} R_{n, k}<\infty$. Then, for arbitrary $\underline{c}=\left(c_{1}, \ldots, c_{n}\right) \in \mathbb{R}^{n}$, with the notation (․2) $-(\mathbb{L} .5)$, we have

$$
\inf _{0<u<1} \Psi_{\underline{c}, k}(u) \leqslant \frac{\mathbb{E}\left[\sum_{i=1}^{n} c_{i}\left(R_{i, k}-\mu\right)\right]}{\Delta} \leqslant \sup _{0<u<1} \Psi_{\underline{c}, k}(u) .
$$

Let $F_{m, a}$ denote the distribution function of the uniform random variable on the interval $\left[a-\frac{1}{m}, a\right]$. If the supremum (infimum) in (ㅁ.6) is attained at some $0<u_{1}<1$, then the upper (lower) bound in (‥6) is attained in the limit by the sequence of parent distribution functions $F_{m}=u_{1} F_{m, a}+\left(1-u_{1}\right) F_{m, b}$ for arbitrary $a<b$. If the supremum (infimum) is attained there in the limit as $u \searrow 0$ ( $u \nearrow 1$ ), then the upper (lower) bound is attained in the limit by any sequence of distribution functions $F_{m}=u_{m} F_{m, a}+\left(1-u_{m}\right) F_{m, b}$ as $m \rightarrow \infty$ and $u_{m} \searrow 0$ ( $u_{m} \nearrow 1$, respectively) whereas $a<b$.

Proof. We start with a useful representation of the expectations of record spacings. For $1 \leqslant i \leqslant n-1$, we have

$$
\begin{aligned}
\mathbb{E}\left(R_{i+1, k}-R_{i, k}\right) & =\int_{-\infty}^{\infty} x G_{i+1, k}(F(d x))-\int_{-\infty}^{\infty} x G_{i, k}(F(d x)) \\
& =\int_{-\infty}^{\infty} x\left(\left(G_{i+1, k}-G_{i, k}\right) \circ F\right)(d x) .
\end{aligned}
$$

Integrating by parts, we obtain

$$
\begin{aligned}
\mathbb{E}\left(R_{i+1, k}-R_{i, k}\right)= & \left.x\left[G_{i+1, k}(F(x))-G_{i, k}(F(x))\right]\right|_{-\infty} ^{\infty} \\
& -\int_{-\infty}^{\infty}\left(G_{i+1, k}(F(x))-G_{i, k}(F(x))\right) d x .
\end{aligned}
$$


Since $\mathbb{E}\left(\left|R_{i, k}\right|\right)<\infty, i=0, \ldots, n$, the first element of the above sum is equal to zero (note that for $x \nearrow \infty$ the difference of distribution functions can be treated as the negative of the difference of respective survival functions). Thus, by (․․), we have

$$
\mathbb{E}\left(R_{i+1, k}-R_{i, k}\right)=\int_{-\infty}^{\infty}[1-F(x)]^{k} \frac{[-k \ln (1-F(x))]^{i}}{i !} d x
$$

We also note that $R_{1, k}=X_{1: k}$ and $\mu=\mathbb{E}\left(\frac{1}{k} \sum_{j=1}^{k} X_{j}\right)=\mathbb{E}\left(\frac{1}{k} \sum_{j=1}^{k} X_{j: k}\right)$. Therefore,

$$
\begin{aligned}
\mathbb{E}\left(R_{n, k}-\mu\right) & =\mathbb{E}\left[\sum_{i=1}^{n-1}\left(R_{i+1, k}-R_{i, k}\right)-\frac{1}{k} \sum_{j=1}^{k}\left(X_{j: k}-X_{1: k}\right)\right] \\
& =\mathbb{E}\left[\sum_{i=1}^{n-1}\left(R_{i+1, k}-R_{i, k}\right)-\frac{1}{k} \sum_{j=2}^{k} \sum_{l=1}^{j-1}\left(X_{l+1: k}-X_{l: k}\right)\right] \\
& =\mathbb{E}\left[\sum_{i=1}^{n-1}\left(R_{i+1, k}-R_{i, k}\right)-\sum_{l=1}^{k-1} \frac{k-l}{k}\left(X_{l+1: k}-X_{l: k}\right)\right] .
\end{aligned}
$$

We further use integral representations of the expected spacings

(1.8) $\mathbb{E}\left(X_{l+1: k}-X_{l: k}\right)=\int_{-\infty}^{\infty}\left(\begin{array}{l}k \\ l\end{array}\right) F^{l}(x)[1-F(x)]^{k-l} d x, \quad l=1, \ldots, k-1$,

due to Pearson [17] (see also Jones and Balakrishnan [1]], formula (3.1)). In particular, we have

$$
\Delta=\mathbb{E}\left|X_{1}-X_{2}\right|=\mathbb{E}\left(X_{2: 2}-X_{1: 2}\right)=2 \int_{-\infty}^{\infty} F(x)[1-F(x)] d x
$$

Combining (ㅍ.7) and (ㄴ.8), we write

$$
\begin{aligned}
\mathbb{E}\left(R_{n, k}-\mu\right)= & \int_{-\infty}^{\infty}\left\{[1-F(x)]^{k} \sum_{i=1}^{n-1} \frac{[-k \ln (1-F(x))]^{i}}{i !}\right. \\
& \left.-\sum_{i=1}^{k-1} \frac{k-i}{k}\left(\begin{array}{c}
k \\
i
\end{array}\right) F^{i}(x)[1-F(x)]^{k-i}\right\} d x \\
= & \int_{-\infty}^{\infty} 2 F(x)[1-F(x)] \Psi_{n, k}(F(x)) d x
\end{aligned}
$$


where

$$
\begin{aligned}
\Psi_{n, k}(u) & =\frac{(1-u)^{k-1}}{2 u} \sum_{i=1}^{n-1} \frac{[-k \ln (1-u)]^{i}}{i !}-\frac{1}{2 u} \sum_{i=1}^{k-1}\left(\begin{array}{c}
k-1 \\
i
\end{array}\right) u^{i}(1-u)^{k-1-i} \\
& =\frac{(1-u)^{k-1}}{2 u} \sum_{i=1}^{n-1} \frac{[-k \ln (1-u)]^{i}}{i !}-\frac{1-(1-u)^{k-1}}{2 u} \\
& =\frac{(1-u)^{k-1} \sum_{i=0}^{n-1} \frac{[-k \ln (1-u)]^{i}}{i !}-1}{2 u}
\end{aligned}
$$

(cf. (‥3)). Finally, for arbitrary $\underline{c} \in \mathbb{R}^{n}$, we get

$$
\mathbb{E}\left[\sum_{i=1}^{n} c_{i}\left(R_{i, k}-\mu\right)\right]=\int_{-\infty}^{\infty} 2 F(x)[1-F(x)] \Psi_{\underline{c}, k}(F(x)) d x,
$$

with

$$
\begin{aligned}
\Psi_{\underline{c}, k}(u) & =\sum_{i=1}^{n} c_{i} \Psi_{i, k}(u)=\frac{(1-u)^{k-1} \sum_{i=1}^{n} c_{i} \sum_{j=0}^{i-1} \frac{[-k \ln (1-u)]^{j}}{j !}-\sum_{i=1}^{n} c_{i}}{2 u} \\
= & \frac{(1-u)^{k-1} \sum_{j=0}^{n-1}\left(\sum_{i=j+1}^{n} c_{i}\right) \frac{[-k \ln (1-u)]^{j}}{j !}-\sum_{i=1}^{n} c_{i}}{2 u}=\frac{\psi_{\underline{c}, k}(u)}{2 u}
\end{aligned}
$$

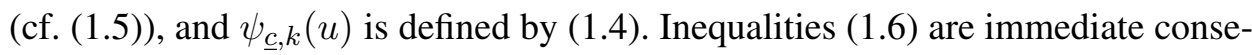
quences of (‥9) and (1.10).

Now we verify the conditions of getting the equality in the right-hand side inequality of (ㄷ.6). The arguments justifying the lower bounds attainability are similar. Suppose first that $\Psi_{\underline{c}, k}\left(u_{1}\right)=\sup _{0<u<1} \Psi_{\underline{c}, k}(u)$ for some $0<u_{1}<1$. The equality

$$
\int_{-\infty}^{\infty} 2 F(x)[1-F(x)] \Psi_{\underline{c}, k}(F(x)) d x=\sup _{0<u<1} \Psi_{\underline{c}, k}(u) \int_{-\infty}^{\infty} 2 F(x)[1-F(x)] d x
$$

holds iff either $F(x)=0$ or $F(x)=1$ or $\Psi_{\underline{c}, k}(F(x))=\Psi_{\underline{c}, k}\left(u_{1}\right)$ for almost all $x \in \mathbb{R}$. The conditions are satisfied by any two-point distribution function

$$
F_{u_{1}}(x)= \begin{cases}0, & x<a \\ u_{1}, & a \leqslant x<b, a<b \\ 1, & x \geqslant b\end{cases}
$$


that assigns probability $u_{1}$ to the smaller point $a$ of its support. We have

$$
\begin{aligned}
\mathbb{E}_{m} R_{i, k}=\int_{-\infty}^{\infty} x G_{i, k}\left(F_{m}(d x)\right) \nearrow \mathbb{E}_{u_{1}} R_{i, k} & =\int_{-\infty}^{\infty} x G_{i, k}\left(F_{u_{1}}(d x)\right)<\infty, \\
\mathbb{E}_{m} X_{1}=\int_{-\infty}^{\infty} x F_{m}(d x) \nearrow \mathbb{E}_{u_{1}} X_{1} & =\int_{-\infty}^{\infty} x F_{u_{1}}(d x)<\infty, \\
\mathbb{E}_{m} X_{i: 2}=\int_{-\infty}^{\infty} x H_{i: 2}\left(F_{m}(d x)\right) \quad \nearrow \mathbb{E}_{u_{1}} X_{i: 2} & =\int_{-\infty}^{\infty} x H_{i: 2}\left(F_{u_{1}}(d x)\right)<\infty,
\end{aligned}
$$

$i=1,2$, as $m \rightarrow \infty$, where $\mathbb{E}_{m}$ and $\mathbb{E}_{u_{1}}$ denote the expectations of various random functions in the cases when $F_{m}$ and $F_{u_{1}}$, respectively, are the parent distribution functions, and $H_{1: 2}(u)=1-(1-u)^{2}$ and $H_{2: 2}(u)=u^{2}, 0<u<1$, are the distribution functions of the minimum and maximum of two i.i.d. standard uniform random variables. Therefore,

$$
\begin{aligned}
\lim _{m \rightarrow \infty} \frac{\mathbb{E}_{m} \sum_{i=1}^{n} c_{i}\left(R_{i, k}-X_{1}\right)}{\mathbb{E}_{m}\left(X_{2: 2}-X_{1: 2}\right)} & =\frac{\mathbb{E}_{u_{1}} \sum_{i=1}^{n} c_{i}\left(R_{i, k}-X_{1}\right)}{\mathbb{E}_{u_{1}}\left(X_{2: 2}-X_{1: 2}\right)} \\
& =\frac{1}{\Delta_{u_{1}}} \int_{-\infty}^{\infty} 2 F_{u_{1}}(x)\left[1-F_{u_{1}}(x)\right] \Psi_{\underline{c}, k}\left(F_{u_{1}}(x)\right) d x \\
& =\sup _{0<u<1} \Psi_{\underline{c}, k}(u)
\end{aligned}
$$

(cf. (‥9) and (ㄷ.10)), as claimed.

Assume now that $\sup _{0<u<1} \Psi_{\underline{c}, k}(u)=\lim _{u \backslash 0} \Psi_{\underline{c}, k}(u)$. Replacing $u_{1}$ of the previous paragraph by arbitrary $0<u<1$, and setting $F_{u, m}=u F_{m, a}+$ $(1-u) F_{m, b}$ with $\mathbb{E}_{u, m}$ standing for the respective expectation functional, we get

$$
\mathbb{E}_{u, m} \sum_{i=1}^{n} c_{i}\left(R_{i, k}-\mu\right) \rightarrow \Psi_{\underline{c}, k}(u) \int_{-\infty}^{\infty} 2 F_{u}(x)\left[1-F_{u}(x)\right] d x .
$$

Replacing fixed $u$ by elements of a sequence $u_{m} \searrow 0$, we finally obtain

$$
\frac{\mathbb{E}_{u_{m}, m} \sum_{i=1}^{n} c_{i}\left(R_{i, k}-\mu\right)}{\Delta_{u_{m}, m}} \nearrow \sup _{0<u<1} \Psi_{\underline{c}, k}(u) .
$$

We proceed in a similar way if $\sup _{0<u<1} \Psi_{\underline{c}, k}(u)=\lim _{u \nearrow 1} \Phi_{\underline{c}, k}(u)$.

REMARK 1.1. It is natural to assume that $c_{n} \neq 0$. Then, for $k=1$ and $n \geqslant 2$, the function $\psi_{\underline{c}, k}$ is unbounded in the left neighborhood of one. It tends either to $+\infty$ or to $-\infty$ there, and the sign coincides with the sign of $b_{n}=c_{n}$. It is clear that $\mathbb{E}\left|X_{1}\right|<\infty$ implies $\Delta=\mathbb{E}\left(X_{2: 2}-X_{1: 2}\right)<\infty$. Nagaraja [16] (see also Arnold et 
al. [1], p. 29) constructed parent distribution functions such that $\mathbb{E}\left|X_{1}\right|<\infty$ and $\mathbb{E} R_{n-1,1}<\infty$, but $\mathbb{E} R_{n, 1}=+\infty$. This justifies the claim that in the case of the first records, there is no finite upper (lower) bound for $\mathbb{E} \sum_{i=1}^{n} c_{i}\left(R_{i, k}-\mu\right) / \Delta$ when $c_{n}>0\left(c_{n}<0\right.$, respectively). However, it may be surprising that we can get an arbitrarily large value (a positive or negative one) even if we restrict ourselves to very simple parent distributions with arbitrarily small supports.

If $k>1$, finiteness of the population mean implies that of all $k$ th records. Note that our bounds are also finite under the assumption.

REMARK 1.2. There are many possibilities of modifying the sequences of distributions attaining the bounds. In the construction $F_{u_{1}, m}=u_{1} F_{m, a}+\left(1-u_{1}\right) F_{m, b}$, the sequences of uniform distribution functions $F_{m, a}, F_{m, b}, m \in \mathbb{N}$, can be substituted with any sequences of continuous distribution functions converging weakly to degenerate ones $F_{a}, F_{b}$ concentrated at $a$ and $b$, respectively. Also, fixed $u_{1}, a, b$ can be replaced by sequences $u_{m}, a_{m}, b_{m}$, with the only restrictions that $u_{n} \rightarrow u_{1}$ and $a_{m}<b_{m}$. Moreover, particular $\Phi_{\underline{c}, k}$ may have multiple extremes. For instance, if $0<u_{1}<\ldots<u_{r}<1$ are some arguments maximizing $\Phi_{c, k}$ (not necessarily all), then the equality in (메) holds for $F=u_{1} F_{a_{0}}+\sum_{i=1}^{r-1}\left(u_{i+1}-u_{i}\right) F_{a_{i}}+$ $\left(1-u_{r}\right) F_{a_{r}}$ for some $a_{0}<\ldots<a_{r}$. In consequence, the upper bound is also attained for any sequence of continuous parent distribution functions tending weakly to the above $(r+1)$-point distribution function. Similar modifications can be used if the extremes of (ㅍ.5) are attained in the limit.

\section{SPECIAL CASES}

In this section we specify sharp bounds of Theorem 1.1 for the most practically important cases of single $k$ th record values and the differences of various $k$ th records. By the theorem, the bounds in the first case coincide with extreme values of functions ([L.3). Note that their derivatives vanish iff

$$
\begin{aligned}
& \chi_{n, k}(u)=2 u^{2} \Psi_{n, k}^{\prime}(u)=u \psi_{n, k}^{\prime}(u)-\psi_{n, k}(u) \\
& =u(1-u)^{k-2}\left[\sum_{i=0}^{n-2} \frac{[-k \ln (1-u)]^{i}}{i !}-(k-1) \frac{[-k \ln (1-u)]^{n-1}}{(n-1) !}\right] \\
& \quad-(1-u)^{k-1} \sum_{i=0}^{n-1} \frac{[-k \ln (1-u)]^{i}}{i !}+1=0 .
\end{aligned}
$$

We do not treat here the first values of $k$ th records $R_{1, k}$, because they coincide with the first order statistics $X_{1: k}$, and the respective evaluations were presented in Kozyra nad Rychlik [14].

Proposition 2.1. Let $X_{1}, X_{2}, \ldots$ be i.i.d. and have a continuous distribution function with finite expectation $\mu=\mathbb{E} X_{1}$ and Gini mean difference $\Delta=$ 
$\mathbb{E}\left|X_{1}-X_{2}\right|$. We also assume that $\mathbb{E}\left|R_{n, k}\right|<\infty$. Then, for various natural $n \geqslant 2$ and $k \geqslant 1$, we have the following sharp bounds:

(i) For $n \geqslant 2$ and $k=1$,

$$
\frac{1}{2}=\Psi_{n, 1}(0+) \leqslant \frac{\mathbb{E}\left(R_{n, 1}-\mu\right)}{\Delta} \leqslant \Psi_{n, 1}(1-)=\infty .
$$

(ii) If $n=k=2$, then

$$
-\frac{1}{2}=\Psi_{2,2}(1-) \leqslant \frac{\mathbb{E}\left(R_{2,2}-\mu\right)}{\Delta} \leqslant \Psi_{2,2}(0+)=\frac{1}{2} .
$$

(iii) For $n \geqslant 3$ and $k=2$,

$$
-\frac{1}{2}=\Psi_{n, 2}(1-) \leqslant \frac{\mathbb{E}\left(R_{n, 2}-\mu\right)}{\Delta} \leqslant \Psi_{n, 2}\left(u_{1}\right)>\frac{1}{2},
$$

where $u_{1} \in(0,1)$ is the unique solution to the particular version of equation (2.1) with $k=2$.

(iv) For $n=2$ and $k \geqslant 3$,

$$
-\frac{1}{2}>\Psi_{2, k}\left(u_{1}\right) \leqslant \frac{\mathbb{E}\left(R_{2, k}-\mu\right)}{\Delta} \leqslant \Psi_{2, k}(0+)=\frac{1}{2},
$$

where $u_{1} \in(0,1)$ is the unique solution to the particular version of (2.1) with $n=2$.

(v) For $k \geqslant 3$ and $n \geqslant 3$,

$$
-\frac{1}{2}>\Psi_{n, k}\left(u_{2}\right) \leqslant \frac{\mathbb{E}\left(R_{n, k}-\mu\right)}{\Delta} \leqslant \Psi_{n, k}\left(u_{1}\right)>\frac{1}{2},
$$

with $0<u_{1}<u_{2}<1$ being the only two solutions to (2.1).

For brevity of presentation, we do not describe precisely attainability conditions. For example, writing that for some parameters $n$ and $k$, the upper bound (or the lower one) is equal to $\Psi_{n, k}\left(u_{1}\right)$ for some uniquely specified $u_{1}$, we refer to Theorem 1.1, where a sequence of mixtures $F_{m}=u_{1} F_{m, a}+\left(1-u_{1}\right) F_{m, b}$ of uniform distributions attaining the bound in the limit is described. Similarly, $\Psi_{n, k}(0+)$ and $\Psi_{n, k}(1-)$ mean that the extreme values of $\Psi_{n, k}$ are attained in the limit as $u \searrow 0$ and $u \nearrow 1$, respectively, and the conditions of attainability can be found again in Theorem 1.1. We also refer to Remark 1.2 for their possible relaxations. We adhere to this convention later on as well.

Table 1 presents numerical values of upper bounds $\Psi_{n, k}\left(u_{1}\right)$ on expectations of $k$ th records for $k=2,8$ and $n=3, \ldots, 11$, and the values of lower bounds $\Psi_{n, 8}\left(u_{2}\right)$ on expectations of eighth records for $n=3, \ldots, 11$. They are accompanied by respective arguments $u_{1}=u_{1}(n, k)$ for which $\Psi_{n, k}$ attain their maxima, and $u_{2}=u_{2}(n, k)$ for which $\Psi_{n, k}$ attain the minima. The lower bounds on the 
TABLE 1. Upper bounds on expectations of $n$th values of second records, and upper and lower bounds on expectations of eighth records for $3 \leqslant n \leqslant 11$.

\begin{tabular}{||r||l|c||c|c||c|c||}
\hline$n$ & $u_{1}(n, 2)$ & $\Psi_{n, 2}\left(u_{1}\right)$ & $u_{1}(n, 8)$ & $\Psi_{n, 8}\left(u_{1}\right)$ & $u_{2}(n, 8)$ & $\Psi_{n, 8}\left(u_{2}\right)$ \\
\hline \hline 3 & 0.53864 & 0.67515 & 0.00612 & 0.50151 & 0.49172 & -0.82907 \\
4 & 0.85953 & 1.27417 & 0.05275 & 0.51740 & 0.63022 & -0.69988 \\
5 & 0.95425 & 2.48879 & 0.12728 & 0.54943 & 0.72995 & -0.63051 \\
6 & 0.98408 & 4.81797 & 0.21163 & 0.59439 & 0.80242 & -0.58866 \\
7 & 0.99425 & 9.23834 & 0.29654 & 0.65089 & 0.85534 & -0.56167 \\
8 & 0.99788 & 17.6289 & 0.37741 & 0.71872 & 0.89407 & -0.54356 \\
9 & 0.99921 & 33.6037 & 0.45206 & 0.79835 & 0.92244 & -0.53108 \\
10 & 0.99971 & 64.1276 & 0.51968 & 0.89066 & 0.94324 & -0.52232 \\
11 & 0.99989 & 122.652 & 0.58017 & 0.99688 & 0.95848 & -0.51611 \\
\hline \hline
\end{tabular}

expectations of second records amount to $\Psi_{n, 2}(1-)=-\frac{1}{2}$. The arguments of the extremes allow us to recover the distributions attaining the bounds. It is obvious that $\Psi_{n, k}\left(u_{1}\right)$ and $\Psi_{n, 8}\left(u_{2}\right)$ increase as $n$ increases from 3 to 11 for both $k=2$ and $k=8$. It is worth noting that $u_{1}(n, k), u_{2}(n, k)$ do so as well.

Let us now evaluate the expectations of differences of $k$ th record values $\mathbb{E}\left(R_{n, k}-R_{m, k}\right), 1 \leqslant m<n$. By Theorem 1.1, the problem boils down to finding the extremes of functions

$$
\begin{aligned}
\Psi_{m, n ; k}(u) & =\Psi_{n, k}(u)-\Psi_{m, k}(u)=\frac{\psi_{n, k}(u)-\psi_{m, k}(u)}{2 u} \\
& =\frac{(1-u)^{k-1}}{2 u} \sum_{i=m}^{n-1} \frac{[-k \ln (1-u)]^{i}}{i !}, \quad 0<u<1 .
\end{aligned}
$$

The local extremes of the functions (if they exist) satisfy the equalities

$$
\begin{aligned}
& \frac{\chi_{m, n ; k}(u)}{(1-u)^{k-2}}=\frac{\chi_{n, k}(u)-\chi_{m, k}(u)}{(1-u)^{k-2}} \\
& \quad=\frac{2 u^{2} \Psi_{m, n ; k}^{\prime}(u)}{(1-u)^{k-2}}=\frac{u\left[\psi_{n, k}^{\prime}(u)-\psi_{m, k}^{\prime}(u)\right]-\left[\psi_{n, k}(u)-\psi_{m, k}(u)\right]}{(1-u)^{k-2}} \\
& =k u \frac{[-k \ln (1-u)]^{m-1}}{(m-1) !}+2 u \sum_{i=m}^{n-2} \frac{[-k \ln (1-u)]^{i}}{i !} \\
& \quad-(k-2) u \frac{[-k \ln (1-u)]^{n-1}}{(n-1) !}-\sum_{i=m}^{n-1} \frac{[-k \ln (1-u)]^{i}}{i !}=0 .
\end{aligned}
$$

PROPOSITION 2.2. Under the assumptions of Proposition 2.1, the following statements hold true: 
(i) If $k=m=1$ and $n \geqslant 2$, then

$$
\frac{1}{2}=\Psi_{1, n ; 1}(0+) \leqslant \frac{\mathbb{E}\left(R_{n, 1}-R_{1,1}\right)}{\Delta} \leqslant \Psi_{1, n ; 1}(1-)=+\infty .
$$

(ii) If $k=1$ and $2 \leqslant m<n$, then

$$
0=\Psi_{m, n ; 1}(0+) \leqslant \frac{\mathbb{E}\left(R_{n, 1}-R_{m, 1}\right)}{\Delta} \leqslant \Psi_{m, n ; 1}(1-)=+\infty .
$$

(iii) If either $k=n=2$ and $m=1$, or $k \geqslant 3, n=2,3$, and $m=1$, then

$$
0=\Psi_{1, n ; k}(1-) \leqslant \frac{\mathbb{E}\left(R_{n, k}-R_{1, k}\right)}{\Delta} \leqslant \Psi_{1, n ; k}(0+)=\frac{k}{2} .
$$

(iv) If $k=2,3, m=1$ and $n \geqslant k+1$, then

$$
0=\Psi_{1, n ; k}(1-) \leqslant \frac{\mathbb{E}\left(R_{n, k}-R_{1, k}\right)}{\Delta} \leqslant \Psi_{1, n ; k}\left(u_{1}\right)>\frac{k}{2},
$$

where $u_{1} \in(0,1)$ is only one solution of equation ([2.3).

(v) For $k=2,3$ with $2 \leqslant m<n$, and for $k \geqslant 4$ with $m \geqslant 2$ and $n=$ $m+1, m+2$, we have

$$
0=\Psi_{m, n ; k}(0+)=\Psi_{m, n ; k}(1-) \leqslant \frac{\mathbb{E}\left(R_{n, k}-R_{m, k}\right)}{\Delta} \leqslant \Psi_{m, n ; k}\left(u_{1}\right)>0,
$$

where $u_{1} \in(0,1)$ is the unique solution to $([2.3)$.

(vi) For $k \geqslant 4, m=1$ and $n \geqslant 4$, equation ([2.3) has either no solutions in $(0,1)$, and then

$$
0=\Psi_{1, n ; k}(1-) \leqslant \frac{\mathbb{E}\left(R_{n, k}-R_{1, k}\right)}{\Delta} \leqslant \Psi_{1, n ; k}(0+)=\frac{k}{2},
$$

or it has two solutions $0<u_{1}<u_{2}<1$, and then

$$
\begin{aligned}
0=\Psi_{1, n ; k}(1-) \leqslant \frac{\mathbb{E}\left(R_{n, k}-R_{1, k}\right)}{\Delta} & \leqslant \max \left\{\frac{k}{2}, \Psi_{1, n ; k}\left(u_{2}\right)\right\} \\
& =\max \left\{\Psi_{1, n ; k}(0+), \Psi_{1, n ; k}\left(u_{2}\right)\right\} .
\end{aligned}
$$

(vii) For all $k \geqslant 4, m \geqslant 2$ and $n \geqslant m+3$, either (2.3) has a unique solution $u_{1}$ in $(0,1)$, and then

$$
0=\Psi_{m, n ; k}(0+)=\Psi_{m, n ; k}(1-) \leqslant \frac{\mathbb{E}\left(R_{n, k}-R_{m, k}\right)}{\Delta} \leqslant \Psi_{m, n ; k}\left(u_{1}\right)>0,
$$

or it has three solutions $u_{1}<u_{2}<u_{3}$ there, and, in consequence,

$$
\begin{aligned}
0=\Psi_{m, n ; k}(0+)=\Psi_{m, n ; k}(1-) & \leqslant \frac{\mathbb{E}\left(R_{n, k}-R_{m, k}\right)}{\Delta} \\
& \leqslant \max \left\{\Psi_{m, n ; k}\left(u_{1}\right), \Psi_{m, n ; k}\left(u_{3}\right)\right\} .
\end{aligned}
$$


The bounds for the most interesting subcase of record spacings $R_{m+1, k}-$ $R_{m, k}$ for particular pairs of parameters $k=m=1, k=1<m, m=1<k$ and $k, m \geqslant 2$ can be immediately concluded from points (i), (ii), (iii), and (v) of Proposition 2.2 , respectively.

TABLE 2. Upper bounds on $\mathbb{E}\left(R_{n, k}-R_{1, k}\right) / \Delta$ for $k=2,3,4$ and $n=4, \ldots, 11$.

\begin{tabular}{||r||c|c||c|c||c|c||}
\hline$n$ & $u_{1}(n, 2)$ & $\Psi_{1, n ; 2}\left(u_{1}\right)$ & $u_{1}(n, 3)$ & $\Psi_{1, n ; 3}\left(u_{1}\right)$ & $u_{1}(n, 4)$ & $\Psi_{1, n ; 4}\left(u_{1}\right)$ \\
\hline \hline 4 & 0.85953 & 1.77417 & 0.24174 & 1.51047 & 0 & 2 \\
5 & 0.95425 & 2.98879 & 0.59908 & 1.64906 & 0 & 2 \\
6 & 0.98408 & 5.31797 & 0.78565 & 1.99633 & 0 & 2 \\
7 & 0.99425 & 9.73834 & 0.88026 & 2.58914 & 0 & 2 \\
8 & 0.99788 & 18.1289 & 0.93089 & 3.50031 & 0.77158 & 2.13923 \\
9 & 0.99921 & 34.1037 & 0.95925 & 4.85335 & 0.84482 & 2.54721 \\
10 & 0.99970 & 64.6276 & 0.97565 & 6.83793 & 0.89233 & 3.11471 \\
11 & 0.99989 & 123.152 & 0.98532 & 9.73634 & 0.92438 & 3.87706 \\
\hline \hline
\end{tabular}

TABLE 3. Upper bounds on $\mathbb{E}\left(R_{n, k}-R_{2, k}\right) / \Delta$ for $k=2,3$ with $n=3, \ldots, 11$, and for $k=10$ with $n=13, \ldots, 21$.

\begin{tabular}{||r||c|c||c|c||c||c|c||}
\hline$n$ & $u_{1}(n, 2)$ & $\Psi_{2, n ; 2}\left(u_{1}\right)$ & $u_{1}(n, 3)$ & $\Psi_{2, n ; 3}\left(u_{1}\right)$ & $n$ & $u_{i}(n, 10)$ & $\Psi_{2, n ; 10}\left(u_{i}\right)$ \\
\hline \hline 3 & 0.79681 & 0.64761 & 0.47471 & 0.54207 & 13 & 0.26010 & 2.08544 \\
4 & 0.90626 & 1.49438 & 0.60992 & 0.96579 & 14 & 0.26018 & 2.08548 \\
5 & 0.96101 & 2.84937 & 0.72476 & 1.38507 & 15 & 0.26021 & 2.08549 \\
6 & 0.98491 & 5.25240 & 0.81886 & 1.87945 & 16 & 0.26021 & 2.08549 \\
7 & 0.99434 & 9.70871 & 0.88772 & 2.54006 & 17 & 0.69271 & 2.13547 \\
8 & 0.99789 & 18.1159 & 0.93242 & 3.48013 & 18 & 0.72874 & 2.24568 \\
9 & 0.99921 & 34.0981 & 0.95956 & 4.84509 & 19 & 0.75943 & 2.37671 \\
10 & 0.99971 & 64.6252 & 0.97571 & 6.83455 & 20 & 0.78605 & 2.52859 \\
11 & 0.99989 & 123.151 & 0.98533 & 9.73495 & 21 & 0.80936 & 2.70201 \\
\hline \hline
\end{tabular}

Table 2 contains numerical values of upper bounds $\Psi_{1, n ; k}\left(u_{1}\right)$ on the expectations of the differences between the $n$th and first values of $k$ th records together with respective arguments $u_{1}=u_{1}(n, k)$ for which $\Psi_{1, n ; k}$ attains its maximum. We examine $k=2,3,4$ and $n=4, \ldots, 11$. For calculating the bounds in cases $k=2,3$, we applied Proposition 2.2(iv). For $k=4$, Proposition 2.2(vi) was used. Then the first subcase of no local extremes appeared for $n=4, \ldots, 7$, and the single local extremes of $\Psi_{1, n ; 4}$ were used for $k=8, \ldots, 11$. As one can expect, the bounds decrease in rows, and increase in columns. The same tendency concerns the arguments providing the maxima. However, it is quite surprising that as $n$ increases, the points attaining the fast increasing maxima approach very close the point one, where the global infima, equal to zero, are attained. 
TABLE 4. Upper bounds on expectations of $k$ th record spacings $\mathbb{E}\left(R_{n+1, k}-R_{n, k}\right) / \Delta$ for $k=2,3,4$ and $n=2, \ldots, 11$.

\begin{tabular}{||r||c|c||c|c||c|c||}
\hline$n$ & $u_{1}(n, 2)$ & $\Psi_{n, n+1 ; 2}\left(u_{1}\right)$ & $u_{1}(n, 3)$ & $\Psi_{n, n+1 ; 3}\left(u_{1}\right)$ & $u_{1}(n, 4)$ & $\Psi_{n, n+1 ; 4}\left(u_{1}\right)$ \\
\hline \hline 2 & 0.79681 & 0.64761 & 0.47471 & 0.54208 & 0.32620 & 0.58475 \\
3 & 0.94048 & 0.94762 & 0.71317 & 0.50558 & 0.54156 & 0.45015 \\
4 & 0.98017 & 1.59328 & 0.83871 & 0.58006 & 0.68538 & 0.43335 \\
5 & 0.99302 & 2.82685 & 0.90731 & 0.72982 & 0.78244 & 0.46358 \\
6 & 0.99748 & 5.15281 & 0.94588 & 0.96486 & 0.84858 & 0.52656 \\
7 & 0.99908 & 9.54491 & 0.96804 & 1.31373 & 0.89404 & 0.62105 \\
8 & 0.99966 & 17.8731 & 0.98097 & 1.82275 & 0.92550 & 0.75117 \\
9 & 0.99988 & 33.7336 & 0.98860 & 2.56139 & 0.94743 & 0.92468 \\
10 & 0.99995 & 64.0592 & 0.99315 & 3.63192 & 0.96278 & 1.15284 \\
11 & 0.99998 & 122.245 & 0.99587 & 5.18426 & 0.97358 & 1.45096 \\
\hline \hline
\end{tabular}

Table 3 presents numerical values of upper bounds $\Psi_{2, n ; k}\left(u_{i}\right)$ on the expectations of differences of $n$th and second values of $k$ th records $\mathbb{E}\left(R_{n, k}-R_{2, k}\right) / \Delta$ with the arguments $u_{i}=u_{i}(n, k), i=1$ or 3 , providing the maxima of respective functions $\Psi_{2, n ; k}$. We considered parameters $k=2,3$ with $n=3, \ldots, 11$, and $k=10$ with $n=13, \ldots, 21$. Conclusions of Proposition 2.2 (v) and (vii) were used for $k=2,3$ and $k=10$, respectively. In the latter case, for $n=13,14$, the function (2.2) has a unique maximum in $(0,1)$, and we use the first statement of Proposition 2.2(vii). Otherwise, it has two local maxima and a minimum between them. However, for $n=15,16$, the global maximum is attained at the first zero of (2.3), and for the remaining $n=17, \ldots, 21$, the last zero provides the global maximum. This explains a significant jump from 0.26021 to 0.69271 in the penultimate column of the table. The behavior of the bounds and parameters describing their attainability conditions are analogous with those in Table 2.

Table 4 presents upper bounds $\Psi_{n, n+1 ; k}\left(u_{1}\right)$ on expectations of $k$ th record spacings $R_{n+1, k}-R_{n, k}$ for $k=2,3,4$ and $n=2, \ldots, 11$, and respective arguments $u_{1}=u_{1}(n, k)$ for which $\Phi_{n, n+1 ; k}$ attains its maximum. They were established by means of Proposition 2.2(v). We observe that except for $k=2$, the bounds first decrease and then increase as $n$ increases. The lower bounds for the differences of records presented in Tables $2-4$ amount to zero.

\section{PROOFS OF PROPOSITIONS 2.1 AND 2.2}

We start with some auxiliary results. The first one is a classic variation diminishing property (VDP, for short) of linear combinations of logarithmic functions used in our studies. 
LEMMA 3.1. The number of sign changes of the linear combination

$$
\sum_{i=1}^{n} a_{i}[-\ln (1-u)]^{\alpha_{i}}, \quad 0<u<1
$$

where $\sum_{i=1}^{n}\left|a_{i}\right|>0$, and $-\infty<\alpha_{1}<\ldots<\alpha_{n}<+\infty$, does not exceed the number of sign changes in the sequence $\left(a_{1}, \ldots, a_{n}\right)$. Moreover, the signs of the function in the right vicinity of zero and the left vicinity of one are identical with the signs of the first and last elements of $\left(a_{1}, \ldots, a_{n}\right)$, respectively.

P r o o f. The first claim can be easily deduced from the analogous property of the family of power functions on the positive half-axis. It asserts that the number of sign changes of the function $x \mapsto \sum_{i=1}^{n} a_{i} x^{\alpha_{i}}$ in $(0,+\infty)$ is not greater than the number of sign changes in $\left(a_{1}, \ldots, a_{n}\right)$ (see, e.g., Karlin and Studden [12], Corollary 1.4.4). Take the strictly increasing reversible function $x=x(u)=-\ln (1-u)$ that transforms $(0,1)$ onto $(0,+\infty)$. This implies that the VDP is inherited by the powers of functions $u \mapsto-\ln (1-u), 0<u<1$. The latter statement of the lemma is trivial.

We also use the following elementary lemma.

LEMMA 3.2. Let $\psi:(a, b) \rightarrow \mathbb{R}, 0 \leqslant a<b$, be a twice differentiable function, $\Psi(x)=\psi(x) / x$, and $\chi(x)=x^{2} \Psi^{\prime}(x)=x \psi^{\prime}(x)-\psi(x)$ with $\chi^{\prime}(x)=x \psi^{\prime \prime}(x)$. We have the following.

(i) If $\psi$ is positive and decreasing, then $\Psi$ decreases.

(ii) If $\psi$ is negative and increasing, then $\Psi$ increases.

(iii) Assume that $\psi$ is convex. Then:

(a) If $\lim _{x / b^{-}} \chi(x) \leqslant 0$, then $\Psi$ is decreasing.

(b) If $\lim _{x \backslash a^{+}} \chi(x) \geqslant 0$, then $\Psi$ is increasing.

(c) If $\lim _{x \backslash a^{+}} \chi(x)<0<\lim _{x / b^{-}} \chi(x)$, then there exists $c \in(a, b)$ such that $\Psi$ decreases on $(a, c]$ and increases on $[c, b)$.

(iv) Suppose that $\psi$ is concave. Then:

(a) If $\lim _{x \backslash a^{+}} \chi(x) \leqslant 0$, then $\Psi$ is decreasing.

(b) If $\lim _{x / b^{-}} \chi(x) \geqslant 0$, then $\Psi$ is increasing.

(c) If $\lim _{x \backslash a^{+}} \chi(x)>0>\lim _{x / b^{-}} \chi(x)$, then there exists $c \in(a, b)$ such that $\Psi$ increases on $(a, c]$ and decreases on $[c, b)$.

The function $\Psi(x)=\psi(x) / x$ represents the slope of the straight line passing through the origin of the real plane, and the graph of $\psi$ at $x$. This is increasing (decreasing) there if the slope is less (greater) than that of the line tangent to $\psi$ at $x$. The function $\psi$ is called starshaped (antistarshaped) if $\psi(x) / x$ is nondecreasing (nonincreasing, respectively). 
Proof of Lemma 3.2. (i) and (ii). By definition, $\operatorname{sgn}\left(\Psi^{\prime}(x)\right)=$ $\operatorname{sgn}(\chi(x))$. Assume that $\psi$ is positive and decreasing. Then $\chi(x)=x \psi^{\prime}(x)-$ $\psi(x)<0$ for all $x \in(a, b), \Psi^{\prime}(x)<0$, and $\Psi$ decreases. Similarly, if $\psi$ is negative and increasing, then $\chi(x)=\psi^{\prime}(x) x-\psi(x)>0$, and hence $\Psi$ increases.

(iii) We have $\chi^{\prime}(x)=x \psi^{\prime \prime}(x)>0$. Under the assumption that $\psi$ is convex, the function $\chi(x)$ increases for all $a<x<b$. Thus, if $\lim _{x>b^{-}} \chi(x) \leqslant 0$, then $\chi(x)<0$ for all $x \in(a, b)$, and so $\Psi$ is decreasing. If $\lim _{x \backslash a^{+}} \chi(x) \geqslant 0$, then $\chi(x)>0$ and $\Psi$ is increasing. Finally, when $\lim _{x \backslash a^{+}} \chi(x)<0<\lim _{x \succ b^{-}} \chi(x)$, then by Darboux's theorem, there exists $a<c<b$ such that $\Psi$ first decreases on $(a, c]$, and then increases on $[c, b)$.

(iv) The proof is analogous to that of (iii).

Pro of of Proposition 2.1. By Theorem 1.1, it suffices to determine

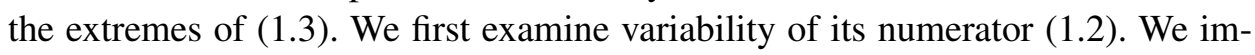
mediately check that $\psi_{n, k}(0+)=0$ for all $k \geqslant 1$ and $n \geqslant 2$ (and for $n=1$ as well, but we do not consider the case here). Also,

$$
\psi_{n, k}(1-)=\left\{\begin{array}{ll}
+\infty, & k=1 \\
-1, & k \geqslant 2,
\end{array} \quad n \geqslant 2\right.
$$

We further have

$$
\psi_{n, k}^{\prime}(u)=(1-u)^{k-2}\left[\sum_{i=0}^{n-2} \frac{[-k \ln (1-u)]^{i}}{i !}-(k-1) \frac{[-k \ln (1-u)]^{n-1}}{(n-1) !}\right] .
$$

If $k=1$, the last term in the square brackets vanishes, and so $\psi_{n, 1}^{\prime}(u)>0$. When $k \geqslant 2$, due to Lemma 3.1, (3.2) is first positive, and then negative. In consequence, for $k \geqslant 2$ the function (․2) is first increasing, and ultimately decreasing. Note that the function is necessarily concave about its maximum, because it is smooth.

The second derivative amounts to

$$
\begin{aligned}
& \psi_{n, k}^{\prime \prime}(u)=(1-u)^{k-3}\left[\sum_{i=0}^{n-3} 2 \frac{[-k \ln (1-u)]^{i}}{i !}\right. \\
& \left.-\left(k^{2}-2\right) \frac{[-k \ln (1-u)]^{n-2}}{(n-2) !}+(k-1)(k-2) \frac{[-k \ln (1-u)]^{n-1}}{(n-1) !}\right]
\end{aligned}
$$

The sum in the brackets does not appear for $n=2$. The middle term is positive for $k=1$, and negative otherwise. The last one vanishes for $k=1$ and 2. Applying Lemma 3.1, we obtain the following conclusions. The function (B.3) is positive for $k=1$ and $n \geqslant 2$, and negative for $k=n=2$. It is first negative, and then positive for $n=2$ and $k \geqslant 3$. For $k=2$ and $n \geqslant 3$, it is consecutively positive, and negative. And finally, for $k, n \geqslant 3$, the sign order is +-+ . Notice that the 
negative part cannot be dropped here, because $\psi_{n, k}$ has a concavity region about its global maximum.

Summing up, we arrived at the following conclusions. If $k=1$ and $n \geqslant 2$, the function (1L.2) convexly increases from 0 at zero to $+\infty$ at one. In all the other cases, it vanishes at both interval end-points. For $k=n=2$, (1L.2) is increasingdecreasing, and concave everywhere. When $k=2$ and $n \geqslant 3$, it is first convex increasing, then concave increasing, and finally concave decreasing. For $n=2$ and $k \geqslant 3$, it is concave increasing on the left, concave decreasing in the center, and convex decreasing on the right. In all the remaining cases $k, n \geqslant 3$, the function is consecutively convex increasing, concave increasing, concave decreasing, and convex decreasing.

Now we are in a position to analyze variability of ([L.3), which is our main task. We start with calculating limit values of ([L.3) at the end-points zero and one. By the l'Hospital rule, for all $n \geqslant 2$,

$$
\begin{aligned}
& \Psi_{n ; k}(0+)=\lim _{u \nearrow 0} \frac{(1-u)^{k-1}-1}{2 u}+\sum_{i=1}^{n-1} \lim _{u \nearrow 0} \frac{[-k \ln (1-u)]^{i}}{2 u i !} \\
= & \lim _{u \nearrow 0} \frac{(1-k)(1-u)^{k-2}}{2}+\sum_{i=0}^{n-2} \lim _{u \nearrow 0} \frac{k[-k \ln (1-u)]^{i}}{2(1-u) i !}=\frac{1-k}{2}+\frac{k}{2}=\frac{1}{2} .
\end{aligned}
$$

Also,

$$
\Psi_{n, k}(1-)=\left\{\begin{array}{ll}
+\infty, & k=1, \\
-\frac{1}{2}, & k \geqslant 2,
\end{array} \quad n \geqslant 2\right.
$$

(cf. (B.d)). Knowing the shapes of (․2.), and using Lemma 3.2 with $\psi(u)=\psi_{n, k}(u)$, $\Psi(u)=2 \Psi_{n, k}(u), \chi(u)=\chi_{n, k}(u)=2 u^{2} \Psi_{n, k}^{\prime}(u)=u \psi_{n, k}^{\prime}(u)-\psi_{n, k}(u), 0<$ $u<1$, we are able to describe monotonicity properties of ([L.3)).

The analysis of the case $k=1<n$ is the simplest one. We have $\chi_{n, 1}(0+)$ $=0$, and the function $\psi_{n, 1}$ convexly increases from $\psi_{n, 1}(0+)=0$ to $\psi_{n, 1}(1-)$ $=+\infty$. By Lemma 3.2(iiib), $\Psi_{n, 1}$ increases from $\Psi_{n, 1}(0+)=\frac{1}{2}$ to $\Psi_{n, 1}(1-)=$ $+\infty$.

We proceed to $k \geqslant 2$ and consider the most sophisticated case with $k, n \geqslant 3$. For the other ones, we refer to some arguments presented here. We assume that $\psi_{n, k}$ is convex increasing on $(0, a)$, concave increasing on $(a, b)$, concave decreasing on $(b, c)$, and convex decreasing on $(c, 1)$ for some $0<a<b<c<1$, and $\psi_{n, k}(d)=0$ for some $b<d<1$. Note that $\chi_{n, k}(0+)=\lim _{u \succ 0}\left[u \psi_{n, k}^{\prime}(u)-\right.$ $\left.\psi_{n, k}(u)\right]=0$. By Lemma 3.2(iiib), $\Psi_{n, k}$ is increasing on $(0, a)$. We have $\chi_{n, k}(a)>0$ because the line tangent to $\psi_{n, k}$ at the inflexion point $a$ runs below the line $\frac{\psi_{n, k}(a)}{a} u$ joining the origin point with $\left(a, \psi_{n, k}(a)\right)$ on $(0, a)$, and above on $(a, 1)$, which means that it has a greater slope. We also have $\chi_{n, k}(b)=-\psi_{n, k}(b)<0$ at the maximum point $b$. Owing to Lemma 3.2(ivc), there is a point $a<u_{1}<b$ such 
that $\Psi_{n, k}$ increases on $\left(a, u_{1}\right)$ and decreases on $\left(u_{1}, b\right)$. By Lemma 3.2(i), $\Psi_{n, k}$ decreases on $(b, d)$. Suppose now that $d<c$. Then $\chi_{n, k}(d)=d \psi_{n, k}^{\prime}(d)<0$, and so $\Psi_{n, k}$ still decreases on $(d, c)$ by Lemma 3.2(iva). Comparing the slopes of straight lines $\psi_{n, k}(c)+\psi_{n, k}^{\prime}(c)(u-c)$ and $\frac{\psi_{n, k}(c)}{c} u$, we conclude that $\chi_{n, k}(c)<0$. We also observe that $\chi_{n, k}(1-)=-\psi_{n, k}(1-)=1>0$. With the use of the last claim of Lemma 3.2(iii), we conclude that $\Psi_{n, k}$ decreases on $\left(c, u_{2}\right)$ and increases on $\left(u_{2}, 1\right)$ for some $c<u_{2}<1$. If $d \geqslant c$, we again recall the relations $\chi_{n, k}(d)<$ $0<\chi_{n, k}(1-)$ and Lemma 3.2(iiic) for deducing that there exists $d<u_{2}<1$ such that $\Psi_{n, k}$ decreases on $\left(d, u_{2}\right)$ and increases on $\left(u_{2}, 1\right)$. Combining the above results, we arrive to the following conclusion: $\Psi_{n, k}$ first increases from $\frac{1}{2}$ at $0+$ to $\Psi_{n, k}\left(u_{1}\right)>\frac{1}{2}$, and then decreases to $\Psi_{n, k}\left(u_{2}\right)<-\frac{1}{2}$, and finally increases to $-\frac{1}{2}$ at $1-$. This implies that the global maximum and minimum are attained at $u_{1}$ and $u_{2}$, respectively, which are the only local extremes of $\Psi_{n, k}$ in $(0,1)$.

If $k=2<n$, the function $\psi_{n, 2}$ does not have a decreasing convex part at the left neighborhood of one. We can just put $c=1>d>b$, and repeat the above reasoning omitting the analysis of the functions on the interval $(c, 1)$ when $d<c$. The case $c \leqslant d<1$ is impossible then. In consequence, we observe that $\Psi_{n, 2}$ increases from $\Psi_{n, 2}(0+)=\frac{1}{2}$ to $\Psi_{n, 2}\left(u_{1}\right)>\frac{1}{2}$, and decreases to $\Psi_{n, 2}(1-)=-\frac{1}{2}$. The global extremes are $\Psi_{n, 2}\left(u_{1}\right)>\frac{1}{2}$ and $\Psi_{n, 2}(1-)=-\frac{1}{2}$.

For $n=2<k, \psi_{2, k}$ is deprived of the increasing convex part on the left. However, then we still have $\chi_{2, k}(0+)=0$, and we can use the argument of Lemma 3.2(iva) to conclude that $\Psi_{2, k}$ is decreasing on $(a, b)$ with $a=0$. Then we repeat the reasoning of the previous paragraph applied to studying the functions $\psi_{n, k}, \chi_{n, k}$, and $\Psi_{n, k}$ on the interval $(b, 1)$. Accordingly, we conclude that $\Psi_{2, k}$ decreases from $\Psi_{2, k}(0+)=\frac{1}{2}$ to $\Psi_{2, k}\left(u_{1}\right)<-\frac{1}{2}$, and increases to $\Psi_{2, k}(1-)=$ $-\frac{1}{2}$. This means that

$$
-\frac{1}{2}>\Psi_{2, k}\left(u_{1}\right) \leqslant \Psi_{2, k}(u)<\Psi_{2, k}(0+)=\frac{1}{2}
$$

For $k=n=2$, we can reduce the arguments as in the two above cases by removing from analysis two convexity intervals of $\psi_{n, k}$ appearing in both the ends of the unit interval. As a result, we see that $\Psi_{2,2}$ decreases from $\Psi_{2,2}(0+)=\frac{1}{2}$ to $\Psi_{2,2}(1-)=-\frac{1}{2}$, which are clearly the extreme values of the function. This completes the proof of Proposition 2.1.

Pro of of Proposition 2.2. The idea is similar to the previous proof. We first analyze the numerator

$$
\psi_{m, n ; k}(u)=(1-u)^{k-1} \sum_{i=m}^{n-1} \frac{[-k \ln (1-u)]^{i}}{i !}
$$

of (․2). We immediately check that $\psi_{m, n ; k}(0+)=0$ for all possible $m, n$, and $k$, 
and $\psi_{m, n ; k}(1-)=+\infty$ when $k=1$, and 0 otherwise. Furthermore,

$$
\begin{aligned}
\psi_{m, n ; k}^{\prime}(u)= & (1-u)^{k-2}\left[k \frac{[-k \ln (1-u)]^{m-1}}{(m-1) !}\right. \\
& \left.+\sum_{i=m}^{n-2} \frac{[-k \ln (1-u)]^{i}}{i !}-(k-1) \frac{[-k \ln (1-u)]^{n-1}}{(n-1) !}\right] .
\end{aligned}
$$

If $k=1$, the last term vanishes, and (B.4) is increasing on the unit interval. By the VDP of Lemma 3.1, the function is first increasing and then decreasing for all $k \geqslant 2$.

The analysis of the second derivative

$$
\begin{aligned}
& \psi_{m, n ; k}^{\prime \prime}(u)=(1-u)^{k-3}\left[k^{2} \frac{[-k \ln (1-u)]^{m-2}}{(m-2) !}\right. \\
& -k(k-3) \frac{[-k \ln (1-u)]^{m-1}}{(m-1) !}+\sum_{i=m}^{n-3} 2 \frac{[-k \ln (1-u)]^{i}}{i !} \\
& \left.-\left(k^{2}-2\right) \frac{[-k \ln (1-u)]^{n-2}}{(n-2) !}+(k-1)(k-2) \frac{[-k \ln (1-u)]^{n-1}}{(n-1) !}\right]
\end{aligned}
$$

is more complex. The coefficient of the first term vanishes for $m=1$, and is positive for $m \geqslant 2$. That of the second one is positive for $k=1,2$, equal to zero for $k=3$, and negative for other $k \geqslant 4$. If $n=m+1, m+2$, the sum is dropped, and its summands are positive otherwise. The penultimate ingredient has a positive coefficient for $k=1$, and a negative one for $k \geqslant 2$. And that of the last one is either zero when $k=1,2$ or positive otherwise.

Applying Lemma 3.1, and taking into account the fact that a smooth function has to be concave about its local maximum, we arrive at the following conclusions. If $k=1$, then (B.5) is positive. Therefore, (3.4) convexly increases from $\psi_{m, n ; k}(0+)=0$ to $\psi_{m, n ; k}(1-)=+\infty$. Otherwise, the function is increasingdecreasing, and vanishes at zero and one.

If $k=2$ and $m=1, n=2$, then $\psi_{m, n ; k}$ is concave in $(0,1)$. If $k=2$ and either $m=1$ with $n \geqslant 3$ or $n>m \geqslant 2$, then (B.5) changes the sign from + to - , which means that (B.4) is first convex increasing, then concave increasing, and finally concave decreasing.

Suppose now that $k=3$. If $m=1$ and $n=2,3$, then (B.5) is negative-positive, and so (B.4) is concave increasing, concave decreasing, and convex decreasing. Otherwise, i.e., for $m=1$ with $n \geqslant 4$, and $n>m \geqslant 2$, the sign sequence of (B.5) is +-+ . This implies that (B.4) is consecutively convex increasing, concave increasing, concave decreasing, and convex decreasing at the right end.

Assume finally that $k \geqslant 4$. Then, for $m=1$ and $n=2,3$, the second derivative (B.5) is negative-positive, and therefore the original function (B.4) is concave 
increasing, concave decreasing, and convex decreasing. If $m=1$ and $n \geqslant 4$, the sign order of the combination coefficients in (B.5)) is -+-+ . For the function (B.5) itself, it may reduce to -+ . Note that in the first case the maximum point of (B.4) can belong to either of the two concavity regions. Consequently, we have three possible behaviors of (B.4). Firstly, it may be concave increasing, concave decreasing, and convex decreasing. Secondly, it may be concave increasing, and, on the region of decrease, it may be consecutively concave, convex, and again concave and convex. The last option is that (B.4) is consecutively concave, convex, and concave on the interval of increase, and concave and convex in the decrease area. If $m \geqslant 2$ and $n=m+1, m+2$, the function (B.5) is first positive, then negative, and eventually positive. It follows that in this case (B.4) is convex increasing, concave increasing and decreasing, and finally convex decreasing. Lastly, for $m \geqslant 2$ and $n \geqslant m+3$, the signs of the combination coefficients are ordered as +-+-+ . The analysis similar to that of the case $k, n \geqslant 4$ with $m=1$ leads to analogous conclusions. We have again three possibilities. The functions are similar, and the only difference is that in each case one should add an interval of convex increase at the beginning.

Now, we proceed to analyzing (2.2). We have

$$
\Psi_{m, n ; k}(0+)= \begin{cases}\frac{k}{2}, & m=1, \\ 0, & m \geqslant 2,\end{cases}
$$

and

$$
\Psi_{m, n ; k}(1-)= \begin{cases}+\infty, & k=1, \\ 0, & k \geqslant 2 .\end{cases}
$$

Also, $\chi_{m, n ; k}(0+)=0$ for all $k, m$, and $n$. This, together with Lemma 3.2(iiib), implies that for $k=1, \Psi_{m, n ; 1}$ strictly increases from $\frac{k}{2}$ when $m=1$, and from 0 for $m \geqslant 2$ at $0+$ to $+\infty$ at $1-$, which gives statements (i) and (ii) of the proposition.

The remaining cases with $k \geqslant 2$ can be treated in much the same way. Functions $\psi_{m, n ; k}$ are first increasing and then decreasing, and tend to zero as the argument tends to zero and one. Respective functions $\chi_{m, n ; k}$ are negative at the maximum point. Functions $\Psi_{m, n ; k}$ are also positive on $(0,1)$, and vanish at the right end-point. Accordingly, zero provides the sharp lower bound for the differences of all $k$ th records with $k \geqslant 2$, and they are attained as the parameter $u$ converges to one. Note that this trivial bound is attained for $m \geqslant 2$ if $u \searrow 0$ as well.

We start with analysis of the most complex case with $k \geqslant 4, m \geqslant 2$ and $n \geqslant m+3$. The first option is that (B.4) is convex, concave, and convex, which implies that the maximum point belongs to the concavity region. We examine it together with another case that (B.4) has two concavity regions, and the maximum is located in the first one. Let $(0, a),(a, b)$, and $(b, 1)$ denote the intervals of convex increase, concave increase, and decrease of the function, respectively. We have 
$\chi_{m, n ; k}(0+)=0<\chi_{m, n ; k}(a)$, and $\chi_{m, n ; k}(b)<0$. By Lemma 3.2 (iiib) and (ivc), the function (2.2) is increasing on $(0, a)$, and increasing-decreasing on $(a, b)$ with a maximum point at $a<u_{1}<b$. By Lemma 3.2(i), it is also decreasing on $(b, 1)$. Therefore, the extreme values of the function are $\Psi_{m, n ; k}(0+)=\Psi_{m, n ; k}(1-)=0$ and $\Psi_{m, n ; k}\left(u_{1}\right)>0$.

Note that the analogous arguments are used for $\Psi_{m, n ; k}$ with parameters $m, n, k$ such that (B.4) is first convex increasing, then concave increasing, and ultimately decreasing, i.e. for $k=2$ with either $m=1$ and $n \geqslant 3$ or $m \geqslant 2$, for $k=3$ with either $m=1, n \geqslant 4$ or $n>m \geqslant 2$, and for $k \geqslant 4$ with $m \geqslant 2$ and $n=m+1$, $m+2$, which cover cases (iv) and (v) of the proposition. The only difference between them is that for $m=1$ the function (․2.) starts from $\frac{k}{2}$, and then the extreme values are $\Psi_{m, n ; k}(1-)=0$ and $\Psi_{m, n ; k}\left(u_{1}\right)>\frac{k}{2}$ (see Proposition 2.2(iv)), and otherwise $\Psi_{m, n ; k}(0+)=0$ is another possibility for the infimum, and then the maximal value $\Psi_{m, n ; k}\left(u_{1}\right)>0$ does not need to exceed $\frac{k}{2}$ (see Proposition 2.2(v)).

Let us come back to $k \geqslant 4, m \geqslant 2$ and $n \geqslant m+3$, and consider the last case that there are two intervals of convex increase $(0, a)$ and $(b, c)$, say, and two intervals of concave increase $(a, b)$ and $(c, d)$. We certainly have $\chi_{m, n ; k}(0+)=0$, $\chi_{m, n ; k}(a)>0$, and $\chi_{m, n ; k}(d)<0$. Lemma 3.2(iiib) implies that $\Psi_{m, n ; k}$ increases on $(0, a)$. Suppose first that $\chi_{m, n ; k}(b) \geqslant 0$. By Lemma 3.2 (ivb) and (iiib), the function (․2.) is increasing on both $(a, b)$ and $(b, c)$. The convexity of $\psi_{m, n ; k}$ on $(b, c)$ implies that $\chi_{m, n ; k}^{\prime}(u)=u \psi_{m, n ; k}^{\prime \prime}(u)>0$ for $b<u<c$, and so $\chi_{m, n ; k}(c)>0$ as well. Lemma 3.2(ivb) assures that there is $c<u_{1}<d$ such that (2.2) is increasing on $\left(c, u_{1}\right)$ and decreasing on $\left(u_{1}, d\right)$. The final decrease of (L.2) on $(d, 1)$ is implied by Lemma 3.2(i). This means that the assumption $\chi_{m, n ; k}(b) \geqslant 0$ leads us to the first statement of Proposition 2.2(vii).

Suppose now that $\chi_{m, n ; k}(b)<0$. Then $\Psi_{m, n ; k}$ is increasing on $\left(0, u_{1}\right)$ and decreasing on $\left(u_{1}, b\right)$ for some $a<u_{1}<b$ by Lemma 3.2 (iiib) and (ivc). By the convexity of $\psi_{m, n ; k}, \chi_{m, n ; k}$ is increasing on $(b, c)$. It may happen that either $\chi_{m, n ; k}(c) \leqslant 0$ or $\chi_{m, n ; k}(c)>0$. Suppose that the first case holds. Then (2.2) decreases on $(b, c),(c, d)$, and $(d, 1)$ by Lemma 3.2 (iiia), (iva) and (i), respectively. Again, we conclude that $\Psi_{m, n ; k}$ has one local maximum in $(0,1)$, and the first claim of Proposition 2.2(vii) holds. The last possibility is that the condition $\chi_{m, n ; k}(b)<0$ is accompanied by $\chi_{m, n ; k}(c)>0$. Then, except for the local maximum at $a<u_{1}<b$, we have a local minimum at $b<u_{2}<c$ by Lemma 3.2(iiic), and another local maximum at $c<u_{3}<d$ by Lemma 3.2(ivc). This is obviously decreasing on $(d, 1)$ by Lemma 3.2(i). Note that $\Psi_{m, n ; k}\left(u_{2}\right)>0$, because $\Psi_{m, n ; k}$ is continuous and positive on $(0,1)$. Accordingly, the latter statement of Proposition 2.2(vii) holds.

The analysis of the penultimate case with $k \geqslant 4, m=1$ and $n \geqslant 4$ is similar, and we merely outline the main steps of the proof. The only differences are that there is no interval on convex increase in the right neighborhood of zero, and 
$\Psi_{1, n ; k}(0+)=\frac{k}{2}$. We can treat together the cases when (2.2) is concave on the whole interval of its increase, whereas the decrease region contains either one or two intervals of convexity. Then $\Psi_{1, n ; k}$ is decreasing on both intervals where $\psi_{1, n ; k}$ increases and decreases by Lemma 3.2 (iva) and (i), and the first claim of Proposition 2.2(vi) is valid. Let us note that in the same way we can treat the cases of Proposition 2.2(iii) and get the respective conclusion.

Suppose now that the interval of increase $(0, d)$, say, contains one region of convexity $(b, c)$, and two regions of concavity $(0, b)$ and $(c, d)$ (we do not use the letter $a$ for the sake of consistency with the previous notation). We have $\chi_{1, n ; k}(0+)=0, \chi_{m, n ; k}(b)<0$, and $\chi_{m, n ; k}(d)<0$. If $\chi_{m, n ; k}(c) \leqslant 0, \Psi_{1, n ; k}$ is decreasing on the whole unit interval by Lemma 3.2 (iva), (iiia), again (iva), and (i). If $\chi_{1, n ; k}(c)>0$, then (2.2) first decreases, then has a unique local minimum at $b<u_{1}<c$, and a unique local maximum at $c<u_{2}<d$, and finally decreases by Lemma 3.2 (ivc), (iiic), again (ivc), and (i). Now, we also have $\Psi_{1, n ; k}\left(u_{1}\right)>$ $\Psi_{1, n ; k}(1-)=0$, but we cannot settle either of two maxima $\Psi_{1, n ; k}(0+)=\frac{k}{2}$, and $\Psi_{1, n ; k}\left(u_{2}\right)$ is greater. This completes the proof of case (vi), and of the whole proposition.

\section{REFERENCES}

[1] B. C. Arnold, N. Balakrishnan, and H. N. Nagaraja, Records, Wiley, New York 1998.

[2] M. Bieniek, Projection mean-variance bounds on expectations of kth record values from restricted families, Comm. Statist. Theory Methods 36 (2007), pp. 679-692.

[3] K. N. Chandler, The distribution and frequency of record values, J. Roy. Statist. Soc. Ser. B 14 (1952), pp. 220-228.

[4] K. Danielak, Distribution-free bounds for expectations of increments of records, J. Statist. Plann. Inference 133 (2005), pp. 239-247.

[5] K. Danielak and M. Z. Raqab, Sharp upper bounds for expectations of kth record increments, Aust. N. Z. J. Stat. 46 (2004), pp. 665-673.

[6] K. Danielak and M. Z. Raqab, Sharp upper bounds on expectations of kth record spacings from restricted families, Statist. Probab. Lett. 69 (2004), pp. 175-187.

[7] W. Dziubdziela and B. Kopociński, Limiting properties of the kth record values, Zastos. Mat. 15 (1976), pp. 187-190.

[8] L. Gajek and A. Okolewski, Projection bounds on expectations of record statistics from restricted families, J. Statist. Plann. Inference 110 (2003), pp. 97-108.

[9] A. Goroncy and T. Rychlik, Lower bounds on the expectations of upper record values, J. Statist. Plann. Inference 141 (2011), pp. 2726-2737.

[10] Z. Grudzień and D. Szynal, On the expected values of kth record values and associated characterizations of distributions, in: Probability and Statistical Decision Theory, Vol. A, F. Konecny, J. Mogyoródy, and W. Wertz (Eds.), Reidel, Dordrecht 1985, pp. 119-127.

[11] M. C. Jones and N. Balakrishnan, How are moments and moments of spacings related to distribution function, J. Statist. Plann. Inference 103 (2002), pp. 377-390.

[12] S. Karlin and W. J. Studden, Tchebysheff Systems: With Applications in Analysis and Statistics, Wiley, New York 1966.

[13] M. Klimczak, Best bounds of kth records from bounded samples, Comm. Statist. Theory Methods 36 (2007), pp. 1451-1464. 
[14] P. M. Kozyra and T. Rychlik, Sharp bounds on the expectations of L-statistics expressed in the Gini mean differences units, Comm. Statist. Theory Methods 46 (2017), pp. 2921-2941.

[15] S. Moriguti, A modification of Schwarz's inequality with applications to distributions, Ann. Math. Statist. 24 (1953), pp. 107-113.

[16] H. N. Nagaraja, On the expected values of record values, Austral. J. Statist. 20 (1978), pp. 176-182.

[17] K. Pearson, Note on Francis Galton's problem, Biometrika 1 (1902), pp. 390-399.

[18] M. Z. Raqab, Bounds based on the greatest convex minorants for moments of record values, Statist. Probab. Lett. 36 (1997), pp. 35-41.

[19] M. Z. Raqab, On the moments of record values, Comm. Statist. Theory Methods 29 (2000), pp. 1631-1647.

[20] M. Z. Raqab, Projection p-norm bounds on the moments of $k$ th record increments, J. Statist. Plann. Inference 124 (2004), pp. 301-315.

[21] M. Z. Raqab, Sharp bounds on expectations of second record increments from decreasing density families, J. Statist. Plann. Inference 137 (2007), pp. 1291-1301.

[22] M. Z. Raqab and T. Rychlik, Sharp bounds for the mean of kth record value, Comm. Statist. Theory Methods 31 (2002), pp. 1927-1937.

[23] M. Z. Raqab and T. Rychlik, Sharp bounds on the expectations of second record values from symmetric populations, Statistics 38 (2004), pp. 49-57.

[24] T. Rychlik, Evaluating improvements of records, Appl. Math. (Warsaw) 24 (1997), pp. 315324.

Paweł Marcin Kozyra

Institute of Mathematics

Polish Academy of Sciences

Śniadeckich 8

00656 Warsaw, Poland

E-mail:pawel_m_kozyra@wp.pl
Tomasz Rychlik Institute of Mathematics Polish Academy of Sciences Śniadeckich 8 00656 Warsaw, Poland E-mail: trychlik@impan.pl

Received on 30.3.2016;

revised version on 19.9.2016 